\subsection{TRANSLATING EVIDENCE INTO POLICY IN LOW AND MIDDLE INCOME COUNTRIES: OPPORTUNITIES AND CHALLENGES}

\author{
Chair: Dr Babu L Verma, India \\ Co Chair: Dr. Ravindra Pandey, India \\ 05-4.1 INDIACLEN EXPERIENCE OF TRANSLATING RESEARCH IN \\ TO POLICY AND PROGRAM
}

doi:10.1136/jech.2011.142976b.54

R M Pandey. * Department of Biostatistics, All India Institute of Medical Sciences, New Delhi, India

IndiaCLEN as one of the seven regional networks of the International Clinical Epidemiology Network (INCLEN), has done number of policy relevant multi centric research in India. The notable among these projects are Assessment of Injection Practices in India; Vit A / IFA supplementation, Several rounds of pulse polio program evaluation, etc. The findings of these research have lead to changes in policy and program in the country. During the session, the challenges, solutions, design, research findings and how these findings lead to the change in policy and program, will be presented.

\section{5-4.2 FROM NCD RESEARCH TO POLICY AND PROGRAM: EXPERIENCE IN PAKISTAN}

doi:10.1136/jech.2011.142976b.55

T Jafar. ${ }^{*}$ Aga Khan University, Karachi, Pakistan

Non-communicable diseases (NCD) have become the leading cause of morbidity and mortality in the population in Pakistan, leading to an estimated loss of US $\$ 3.5$ billion annually just from productive life years lost. The prevalence of hypertension has increased exponentially, with trends data suggesting reversal of the social gradient over the last decade tilting a heavier burden on the disadvantaged population. Pakistan has the sixth highest number of people in the world with diabetes, every fourth adult is overweight and the condition is rapidly escalating in children, cigarettes are cheap, antismoking regulations are poorly enforced, and mixed public-private healthcare system provide suboptimal care. Unfortunately, the unstable geo-political situation in Pakistan has retarded economic and social development in the country. Despite these challenges, evidence also indicates that reconfiguration of the health systems framework using the existing physician and non-physician workforce can yield promising results with economically efficient, affordable, and sustainable programs for NCD prevention and control. A National Commission on NCDs has also been proposed for recommendations on programs and policies. Successful implementation of these efforts is urgent and essential for translation of evidence to improved NCD outcomes in this high risk population.

\section{5-4.3 MANAGEMENT OF NON-COMMUNICABLE DISEASES IN THE GAUTENG PROVINCE IN SOUTH AFRICA}

doi:10.1136/jech.2011.142976b.56

\footnotetext{
1,2D Basu. * ${ }^{1}$ Department of Community Health, Charlotte Maxeke Johannesburg Academic Hospital and University of the Witwatersrand, Johannesburg, South Africa; ${ }^{2}$ Gauteng Department of Health, Johannesburg, South Africa
}

Background In South Africa, the burden of Non-communicable diseases (NCD) is staggering (37\% in terms of Initial Burden of Disease, 2000 report). The NCD include cardiovascular disease, stroke, diabetes mellitus, asthma and respiratory diseases, epilepsy and cancer. In line with its vision, the Public Health Directorate have decided to be proactive in managing this problem by setting up a surveillance system based on WHO stepwise model for improvement of management of the NCD at the provincial health facilities. Objectives The purpose of this project is to improve the burden of NCD in the Province. The objectives of this project include evaluation of health facility data on NCD; Development of an intervention strategy for improving NCD in the Health Districts; Implementation of the intervention plan; and Setting-up a surveillance site for the continuous monitoring and evaluation of noncommunicable disease in this District.

Results The study found a significant burden of NCD (such cardiometabolic diseases, cancer) in the Province in comparison to the rest of South Africa and other countries of Africa. This burden in addition to burden of communicable diseases (such as tuberculosis and HIV/AIDS) would require significant resource allocation for effective management. An integrated approach to healthcare is necessary to address this.

\section{5-4.4 TRANSLATING EVIDENCE INTO POLICY IN CHINA: OPPORTUNITIES AND CHALLENGES}

doi:10.1136/jech.2011.142976b.57

J Wang.* Department of Internal Medicine, Zhongshan Hospital, Fudan University, Shanghai, China

Research and evidence are critical for informing policies and practices in support of population healthcare and equity. In the past 2 decades, ChinaCLEN's roles and responsibilities promote evidence-based policy-making in China. Evidence-based policy has become a major part of governments' approaches for policy making and the machinery of government. In this presentation I will mainly focus on the transformation of national government healthy policy and strategies according to the expansion of evidence-based medicine in China, as well as the opportunities and challenges in the future. During the symposium following aspects in China will be discussed: 1 . Organisation on the Evidence Based Health Care; 2. Impacts on Government Healthy Policy Decision-Making; 3. The prioritised research agenda special focus on clinical research identified and advocated for key research needs in 2011-2015; 4. Contributions on evidence based public health safety policy and practice; 5 . Standards for scientific review of the clinical efficacy of Chinese traditional medicine; 6 . Establishing integration system of traditional Chinese medicine and clinical research; 7. Improving accessibility and availability of sound evidence; and, 8. Future Opportunities and Challenges.

\subsection{SOCIAL POLICY}

\section{Chair: Dr Patricia Buffler, USA 05-5.1 DOES HEALTHCARE SPENDING IMPROVE HEALTH OUTCOMES AND REDUCE HEALTH INEQUALITIES?}

doi:10.1136/jech.2011.142976b.58

D Lemon, ${ }^{*} \mathrm{R}$ Khan. Dorset PCT, Dorset, UK

Health systems around the world are struggling to cope with increasing healthcare costs and rising demand due to an ageing population. There have been several financial initiatives in the NHS to motivate improved performance.

The aim of this study is to examine the link between healthcare expenditure in both primary and secondary care and health outcomes and whether this relationship varies with levels of deprivation.

The study is done in Dorset PCT, which has one of the highest proportion of over 75 year olds in England. The dataset in the study is 